\title{
Distribution Systems State Estimation Using Sparsified Voltage Profile
}

M. Majidi ${ }^{\text {a }}$, M. Etezadi-Amoli ${ }^{* a}$, H.Livani ${ }^{\text {a }}$, M. S. Fadali ${ }^{\text {a }}$

${ }^{a}$ Department of Electrical and Biomedical Engineering, University of Nevada, Reno, USA

* Corresponding author address: 1664 N. Virginia Street, M/S 260, Reno, NV 89557, USA

Telephone number: 775-784-6090

Fax number: 775-784-6627

Email address: etezadi@unr.edu

Abstract- This paper exploits an electrical characteristic of distribution networks to cast the state estimation problem into a sparse vector recovery problem. In distribution networks, voltage differences between two buses of each line segment are much smaller than the infeed bus voltage. Therefore, the voltage profile signal can be sparsified with a difference transformation and recovered from only a few micro-phasor measurement units ( $\mu$ PMUs) using compressive sensing (CS) and $\ell_{1}$ regularization. The effectiveness of the proposed algorithm is verified through the simulation results of a standard unbalanced distribution network, the IEEE 123bus system, under different operation conditions. The method accurately estimates system states even with multiple bad current measurements. It also detects, identifies, and corrects bad voltage measurements. In addition, a problem of binary integer linear programming is solved to obtain and optimally place the minimum number of $\mu$ PMUs necessary to provide a unique solution for the proposed state estimation formulation.

Keywords- State estimation; distribution networks; micro-phasor measurement units ( $\mu$ PMUs); $\ell_{1}$-norm minimization; compressive sensing (CS); bad data processing.

\section{INTRODUCTION}

\subsection{Literature Review}

The proliferation of distribution energy resources (DER) e.g. electric vehicles, 
distributed generators (DGs), and demand response necessitates visibility and situational awareness in smart distribution networks. Unlike legacy radial networks with one-way power flow, smart distribution networks with multiple sources contain notable variability and uncertainties that must be continuously observed and actively managed [1]. Active management (AM) is provided in distribution networks if a distribution management system (DMS) with various functionalities, such as state estimation (SE), fault detection, isolation, and restoration (FDIR), and volt/var control, are enabled for system operation. The performance of several DMS applications relies on the quality of the state estimates provided by the distribution system state estimation (DSSE) application. DSSE is a subject of several contributions in the literature since early nineties. In [2], DSSE is formulated by Kirchhoff's laws and load data is estimated from statistical models. Baran and Kelley [3] introduce the weighted least-squares (WLS)based DSSE algorithm whose performance relies heavily on load allocation accuracy. In [4], they proposed an unbalanced three-phase SE approach using branch current measurements. Lu, et al. [5] developed a two-step current-based DSSE in which distribution transformer loadings are forecasted first and nonlinear load flow formulations are subsequently solved by Newton's method. In [6], [7], the current-based DSSE is examined by decoupling the gain matrix in the state estimation process. In [8], load allocation challenges are discussed by implementing a state estimator in a real distribution network.

Unlike transmission networks, supervisory control and data acquisition (SCADA) measurements are not widely available in distribution networks. This results in low measurement redundancy and is the major obstacle for improving the quality of state estimation in distribution networks. Most DSSE algorithms complement the limited set 
of available real-time measurements with pseudo-measurements, usually obtained from load forecast studies. Li [9] statistically modeled the loads based on their time-varying characteristic and weather conditions. Ghosh, et al. [10] used a non-normal distribution of load variations to develop a probabilistic load model. Recently, artificial intelligence (AI) methods e.g. artificial neural networks (ANNs), nonlinear autoregressive techniques, and fuzzy logic were used to forecast the load data in DSSE algorithms [11]-[13]. However, high uncertainties in power injection patterns and lack of synchronization between the predicted loads and real-time measurements limit the practical usefulness of the load allocation methods for DSSE.

More recently, intelligent meter data have been also used to improve the measurement redundancy and, consequently, improve the performance of state estimators [14]. However, smart meters (SMs) have a number of problems affecting the performance of DSSE methods. SMs generally record the sampling time of their measurements with some error. These measurements cannot be simultaneously transmitted due to bandwidth limitations [14]. In addition, different SMs send their measured data to the monitoring center with different time delays [15]. Therefore, SMs often provide unsynchronized measurements in such a way that sampling times of some measurements may vary significantly under some operating conditions. The error due to lack of synchronization is compensated for by adding an error term to the measurement device error in the WLS-based DSSE methods [14]. However, this approach makes the SM measurements less reliable and consequently affects DSSE performance. In addition, the high cost of deploying SMs and their corresponding infrastructures for a huge number of nodes prevent distribution companies from making their networks fully observable. In [16], the Hamilton cycle theory is used to estimate the states in the 
distribution networks. It is assumed that the magnitudes and angles of voltages and currents are synchronously measured by smart meters in some buses of the distribution networks.

\subsection{Contribution}

A more efficient and more accurate DSSE method using a few low-cost and ultrahigh-resolution micro-phasor measurement units ( $\mu \mathrm{PMUs})$ is proposed here. $\mu \mathrm{PMU}$ is a new technology developed and certified at the University of California in collaboration with the Power Standards Lab (PSL) and Lawrence Berkeley National Lab (LBNL), funded by the U.S Department of Energy. The device has been installed and tested in two nodes of a real distribution network and will be installed in hundreds of buses at partner utilities [17].

This paper proposes an original application of compressive sensing (CS) to DSSE that drastically differs from the usual WLS-based state estimation. It is inspired by the underlying hypothesis that voltage differences between any two buses of every line segment are small and negligible compared with the infeed point voltage in distribution networks. Therefore, the voltage profile is transformed to a sparse domain using a difference transformation and is recovered from far fewer measurements than those required for WLS and other conventional techniques. The use of CS for distribution network fault location and power system state estimation has been presented in [18][21]. This paper extends the application of CS to distribution system state estimation and enhances DMS capabilities. In addition, a problem of binary integer linear programming is solved to obtain and optimally place the minimum number of $\mu$ PMUs necessary to provide a unique solution for the proposed state estimation formulation.

The rest of the paper is organized as follows. Section II presents the state estimation equations solved by $\mathrm{CS}$ and $\ell_{1}$ regularization based techniques and proposes a bad data 
detection, identification, and correction procedure. Section III demonstrates the simulation results of the proposed DSSE algorithm in different operation scenarios. The effects of the noisy measurements, bad data, DGs, and the meshed operation mode on the performance of the proposed method are examined. Section IV compares the proposed method with other methods and discusses the advantages of our approach. Our conclusion is presented in Section V.

\section{STATE ESTIMATION FORMULATION}

$\mu$ PMUs measure current flows through incident branches to buses and bus voltages synchronously [22]. The measured phasors are expressed in terms of the system states by

$$
Z_{0}=H_{0} x_{0}+v_{0}
$$

where $Z_{0} \in R^{m_{0}}, m_{0}=p+q$, is the measurement vector including $p$ three-phase voltage and $q$ three-phase current measurements, $x_{0} \in R^{n_{0}}$ is the three-phase state vector, $v_{0} \in R^{m_{0}}$ is the measurement error vector. $H_{0} \in R^{m_{0} \times n_{0}}$ is the constant measurement Jacobian matrix

$$
H_{0}=\left[\begin{array}{c}
I I \\
y A+y_{s}
\end{array}\right]
$$

where $I I \in R^{p \times n_{0}}$ is the three-phase representation of the voltage measurement-bus incident matrix, $y \in R^{q \times q}$ is the three-phase series admittance matrix, $A \in R^{q \times n_{0}}$ is the three-phase representation of current measurement-bus incident matrix, and $y_{s} \in R^{q \times n_{0}}$ is the three-phase shunt admittance matrix. For example, the equivalent PI circuit of a 3bus distribution system is shown in Fig. 1. Note that line 2-3 has only two phases. 


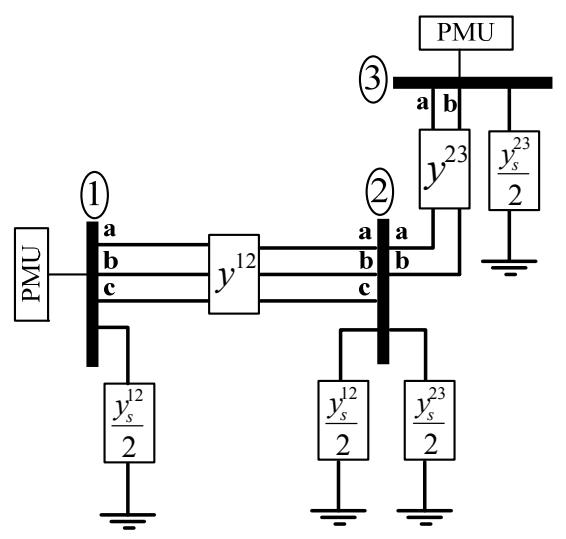

Fig. 1. 3-bus distribution network

where:

$$
\begin{aligned}
y^{12} & =\left[\begin{array}{lll}
y_{a a}^{12} & y_{a b}^{12} & y_{a c}^{12} \\
y_{b a}^{12} & y_{b b}^{12} & y_{b c}^{12} \\
y_{c a}^{12} & y_{c b}^{12} & y_{c c}^{12}
\end{array}\right], y^{23}=\left[\begin{array}{ll}
y_{a a}^{23} & y_{a b}^{23} \\
y_{b a}^{23} & y_{b b}^{23}
\end{array}\right], \\
y_{s}^{12} & =\left[\begin{array}{lll}
y_{s, a a}^{12} & y_{s, a b}^{12} & y_{s, a c}^{12} \\
y_{s, b a}^{12} & y_{s, b b}^{12} & y_{s, b c}^{12} \\
y_{s, c a}^{12} & y_{s, c b}^{12} & y_{s, c c}^{12}
\end{array}\right], y_{s}^{23}=\left[\begin{array}{ll}
y_{s, a a}^{23} & y_{s, a b}^{23} \\
y_{s, b a}^{23} & y_{s, b b}^{23}
\end{array}\right] .
\end{aligned}
$$

If two $\mu$ PMUs are installed at buses 1 and 3, we have

$$
\begin{aligned}
& Z_{0}=\left[\begin{array}{llllllllll}
V_{a}^{1} & V_{b}^{1} & V_{c}^{1} & V_{a}^{3} & V_{b}^{3} & I_{a}^{12} & I_{b}^{12} & I_{c}^{12} & I_{a}^{32} & I_{b}^{32}
\end{array}\right]^{T} \\
& x_{0}=\left[\begin{array}{llllllll}
V_{a}^{1} & V_{b}^{1} & V_{c}^{1} & V_{a}^{2} & V_{b}^{2} & V_{c}^{2} & V_{a}^{3} & V_{b}^{3}
\end{array}\right]^{T} \\
& I I=\left[\begin{array}{ccc}
I_{3} & O_{3 \times 3} & O_{3 \times 2} \\
O_{2 \times 3} & O_{2 \times 3} & I_{2}
\end{array}\right] \\
& A=\left[\begin{array}{ccc}
I_{3} & -I_{3} & O_{3 \times 2} \\
O_{2 \times 3} & -I_{2} \vdots O_{2 \times 1} & I_{2}
\end{array}\right] \\
& y=\left[\begin{array}{cc}
y^{12} & O_{3 \times 2} \\
O_{2 \times 3} & y^{23}
\end{array}\right], y_{s}=\left[\begin{array}{lll}
y_{s}^{12} & O_{3 \times 3} & O_{3 \times 2} \\
O_{2 \times 3} & O_{2 \times 3} & y_{s}^{23}
\end{array}\right] \\
& y A+y_{s}=\left[\begin{array}{ccc}
y^{12}+y_{s}^{12} & -y^{12} & O_{3 \times 2} \\
O_{2 \times 3} & -y^{23}: O_{2 \times 1} & y^{23}+y_{s}^{23}
\end{array}\right]
\end{aligned}
$$

where $O_{M \times N}$ is an $M$-by- $N$ matrix of zeros and $I_{N}$ is an $N$-by- $N$ identity matrix.

Rewriting (1) in rectangular coordinates gives

$$
\left[\operatorname{Re}\left\{Z_{0}\right\}+j \operatorname{Im}\left\{Z_{0}\right\}\right]=\left[\operatorname{Re}\left\{H_{0}\right\}+j \operatorname{Im}\left\{H_{0}\right\}\right] \cdot\left[\operatorname{Re}\left\{x_{0}\right\}+j \operatorname{Im}\left\{x_{0}\right\}\right]+\left[\operatorname{Re}\left\{v_{0}\right\}+j \operatorname{Im}\left\{v_{0}\right\}\right]
$$


Separating the real and imaginary terms, (3) is rewritten in matrix form

$$
\begin{gathered}
\overbrace{\left[\begin{array}{l}
\operatorname{Re}\left\{Z_{0}\right\} \\
\operatorname{Im}\left\{Z_{0}\right\}
\end{array}\right]}^{Z}=\overbrace{\left[\begin{array}{cc}
\operatorname{Re}\left\{H_{0}\right\} & -\operatorname{Im}\left\{H_{0}\right\} \\
\operatorname{Im}\left\{H_{0}\right\} & \operatorname{Re}\left\{H_{0}\right\}
\end{array}\right]}^{H\left[\begin{array}{l}
\operatorname{Re}\left\{x_{0}\right\} \\
\operatorname{Im}\left\{x_{0}\right\}
\end{array}\right]}+\overbrace{\left[\begin{array}{l}
\operatorname{Re}\left\{v_{0}\right\} \\
\operatorname{Im}\left\{v_{0}\right\}
\end{array}\right]}^{v} \\
Z=\text { H.x } x
\end{gathered}
$$

where $\quad Z \in R^{m}, \quad H \in R^{m \times n}, \quad x \in R^{n}, \quad$ and $\quad v \in R^{m} \quad\left(m=2 m_{0}, \quad n=2 n_{0}\right)$ are representation of $Z_{0}, H_{0}, x_{0}$, and $v_{0}$ in rectangular coordinates, respectively.

\section{$2.1 \ell_{2}$ and $\ell_{1}$ regularizations in DSSE}

When the number of measurements $(m)$ is much larger than the number of system states $(n)$ in (4), the matrix $(H)$ is likely to be full rank and the vector $(x)$ can be calculated by least-squares error minimization. However, over-fitting occurs in the least-squares technique if $m$ is slightly larger than $n$. To avoid over-fitting, $\ell_{2}$ or Tikhonov regularization is used to solve (4) by [23]

$$
\min \|H \cdot x-Z\|_{2}^{2}+\lambda\|x\|_{2}^{2}
$$

where $\|\cdot\|_{2}$ denotes the $\ell_{2}$ norm operator and $\lambda>0$ is the regularization factor. The solution of the $\ell_{2}$-regularized least-squares problem is

$$
x_{\ell 2}=\left(H^{T} H+\lambda I\right)^{-1} H^{T} Z
$$

When $m<n$, (4) is an underdetermined system of equations with infinite solutions. However, if there is a priori knowledge that $x$ is a sparse vector most of whose entries are zero, it can be recovered with a few measurements by solving the $\ell_{1}$-regularized least-squares problem [24]

$$
\min \|H \cdot x-Z\|_{2}^{2}+\lambda\|x\|_{1}
$$

where $\|.\|_{1}$ denotes the $\ell_{1}$ norm. Unlike Tikhonov regularization, the $\ell_{1}$-regularized least-squares technique yields a vector $(x)$ which is not a linear function of the vector $(Z)$. In addition, the limiting point of (7) is the minimum $\ell_{1}$ norm solution that satisfies 
$H^{T}(H . x-Z)=0$. The optimal solution of (7) is obtained for a finite value of $\lambda$

$$
\lambda \leq\left\|2 H^{T} Z\right\|_{\infty}=\lambda_{\max }
$$

where $\|.\|_{\infty}$ denotes the $\ell_{\infty}$ norm. Additionally, the family of solutions or the regularization path of (7) is fitted by a piecewise linear curve

$$
\begin{aligned}
& x_{\ell 1}=\overbrace{\left(\frac{x_{k}-x_{k+1}}{\lambda_{k}-\lambda_{k+1}}\right)}^{a} \lambda+\overbrace{\frac{\lambda_{k} x_{k+1}-\lambda_{k+1} x_{k}}{\lambda_{k}-\lambda_{k+1}}}^{b} \\
& \lambda_{k+1} \leq \lambda \leq \lambda_{k}, k=1, \ldots, i-1 \\
& 0=\lambda_{i} \leq \cdots \leq \lambda_{1}=\lambda_{\max }
\end{aligned}
$$

where $\mathrm{x}_{\mathrm{k}}$ is a solution of the (7) with $\lambda=\lambda_{\mathrm{k}}$. Parameters $a$ and $b$ are computed by selecting any two values $\lambda_{k}$ and $\lambda_{k+1}$, with $0<\lambda_{k+1}<\lambda_{k}<\lambda_{\max }$, and calculating the corresponding solutions $x_{k}$ and $x_{k+1}$ using (7). Then we vary $\lambda$ in the range $\left(0, \lambda_{\max }\right]$ and substitute the $x_{\ell 1}$ value obtained from (9a) in the objective function of (7). The $\lambda$ value that minimizes the objective function is selected.

\subsection{Compressive Sensing Approach}

When $(x)$ is not sparse but is compressible, it can be converted to a sparse vector by [25]

$$
x_{s}=S \cdot x
$$

where $x_{s} \in R^{n}$ is the sparse representation of the vector $x$ in the transform domain and $S \in R^{n \times n}$ is a sparsifying transform matrix. When $S$ is nonsingular, the vector $(x)$ is obtained by $x=S^{-1} \cdot x_{s}$ and substituted in (4b) to form

$$
Z=\psi \cdot x_{s}+v
$$

where $\psi=H . S^{-1}$. Equation (11) is solved by

$$
\min \left\|\psi \cdot x_{s}-Z\right\|_{2}^{2}+\lambda\left\|x_{s}\right\|_{1}
$$

The solution of (12) $x_{s}^{*}$ yields $x^{*}=S^{-1} \cdot x_{s}^{*}$ as the solution of (7). Unlike (5), the 
objective function in the $\ell_{1}$-regularized least-squares problems (7) and (12) is convex but not differentiable and must be computed numerically. Researchers have proposed different methods for solving this problem [24], [26], [27]. In this paper, we adopt an interior-point method that can efficiently solve a large-scale system of equations with a million states in a few seconds [24]. This method is more efficient than the techniques proposed in [26] and [27] for distribution networks containing of many buses. We briefly review the interior-point method by describing the duality problem, suboptimality bound, and truncated Newton interior-point method.

\subsubsection{Duality theorem and sub-optimality bound}

The Lagrange dual of (12) is formulated by defining [24]

$$
\min r^{T} \cdot r+\lambda_{i}\left\|x_{s}\right\|_{1} \quad \text { subject to } \quad r=\psi \cdot x_{s}-Z
$$

The dual problem of (13) is a convex optimization problem

$$
\max G(w)=-0.25 w^{T} w-w^{T} Z \text { subject to }\left|\left(\psi^{T} . w\right)_{i}\right| \leq \lambda_{i}, i=1, \ldots, m
$$

where $w \in R^{m}$ is the dual variable vector and $w_{i}$ corresponds to $r_{i}=\left(\psi \cdot x_{s}-Z\right)_{i}$. Based on the strong duality theorem, the primal and dual problems have identical optimal solutions.

In addition, a computed bound on the sub-optimality of $x_{s}$ can be obtained at a dual feasible point

$$
w=2 t\left(\psi \cdot x_{s}-Z\right), \quad t=\min \left\{\frac{\lambda}{\left|2\left(\left(\psi^{T} \psi x_{s}\right)_{i}-2 Z_{i}\right)\right|}\right\}, i=1, \ldots, m
$$

Since $w$ is a dual feasible point, $G(w)$ is a lower bound on $\alpha,(G(w) \leq \alpha)$ and $\alpha$ is the sub-optimal value of (12).

The primal objective value of $x_{s}$ in (12) is subtracted from $G(w)$ to provide the duality gap function $(\varphi)$

$$
\varphi=\left\|\psi \cdot x_{s}-Z\right\|_{2}^{2}+\lambda\left\|x_{s}\right\|_{1}-G(w)
$$


Based on the strong duality theorem, the optimal solution $\left(x_{s}^{*}\right)$ is obtained when the duality gap is zero under a constraint qualification condition. Based on the linearity constraint qualification, since the inequality constraints in (14) are affine functions, no other conditions are needed.

\subsubsection{Truncated Newton interior-point method}

Equation (12) can be reformulated to a convex quadratic problem (QP) with the inequality constraints

$$
\min \left\|\psi \cdot x_{s}-Z\right\|_{2}^{2}+\lambda \sum_{i=1}^{n} h_{i} \text { subject to }-h_{i} \leq x_{s, i} \leq h_{i}, i=1, \ldots, n
$$

The QP (17) is solved by an interior-point technique. A logarithmic barrier function is derived from the linear inequality constraints in (17)

$$
\tau\left(x_{s}, h\right)=-\sum_{i=1}^{n} \log \left(x_{s, i}+h_{i}\right)-\sum_{i=1}^{n} \log \left(h_{i}-x_{s, i}\right)
$$

The inequality constraints $\left|x_{s, i}\right|<h_{i}, i=1, \ldots, n$ yield a central path including point $\left(x_{s}^{*}(u), h^{*}(u)\right)$ that uniquely minimizes the convex function

$$
\tau_{u}\left(x_{s}, h\right)=u\left\|\psi \cdot x_{s}-Z\right\|_{2}^{2}+u \sum_{i=1}^{n} \lambda h_{i}+\tau\left(x_{s}, h\right)
$$

where $0 \leq u \leq \infty$. Since $2 n / u$ is the sub-optimal bound on $\left(x_{s}^{*}(u), h^{*}(u)\right)$, the optimal solution is on the central path. The dual feasible solution of any point $\left(x_{s}^{*}(u), h^{*}(u)\right)$ on the central path is $w^{*}(u)=2\left(\psi \cdot x_{s}^{*}(u)-Z\right)$.

In the primal interior-point method, $\left(x_{S}^{*}(u), h^{*}(u)\right)$ is sequentially calculated for $u=u_{0}, \delta u_{0}, \delta^{2} u_{0}, \ldots$ until $2 n / u \leq \varepsilon$, where $2 \leq \delta \leq 50$ and $\varepsilon$ is the duality gap threshold. The $\varepsilon$-sub-optimality of $\left(x_{s}^{*}(u), h^{*}(u)\right)$ is then ensured. In the primal barrier method (20) is solved to determine the search direction for $\tau_{u}$ minimization

$$
\gamma\left[\begin{array}{c}
\Delta x_{s} \\
\Delta h
\end{array}\right]=-g
$$


where $\gamma=\nabla^{2} \tau_{u}\left(x_{s}, h\right) \in R^{2 n \times 2 n}$ is the Hessian and $g=\nabla \tau_{u}\left(x_{s}, h\right) \in R^{2 n}$ is the gradient at $\left(x_{s}, h\right)$. To reduce the computational load of solving (20) with a large number of states, the preconditioned conjugate gradient (PCG) technique is used to calculate the search direction [24]. This iterative solving process is so-called the truncated Newton method.

In the primal barrier method, the term $u$ is constant in the $\tau_{u}$ minimization process. However, updating $u$ with respect to the duality gap in each iteration improves the rate of convergence [24]. The states estimation is carried out by solving the $\ell_{1}$-regularized least-squares problem of (12) using the truncated Newton interior-point algorithm as [24]:

1. Define a relative tolerance $\varepsilon_{r}>0$ and initialize $u=1 / \lambda, x_{s}=O_{n \times 1}, h=\mathbf{1}^{n \times 1}$.

2. Solve (20) approximately to find the search direction $\left(\Delta x_{s}, \Delta h\right)$.

3. Calculate $t$ in (15) and update the iterate with $\left(x_{s}, h\right)=\left(x_{s}, h\right)+t\left(\Delta x_{s}, \Delta h\right)$.

4. Compute the dual feasible point $w$ of (15).

5. Construct the duality gap $\varphi$ of (16).

6. Stop if $\varphi / G(w) \leq \varepsilon_{r}$, otherwise update $u$ and go to step 2 .

\subsection{Sparsifying Voltage Profile in Distribution Networks}

Our extensive simulation studies on several standard networks with a large range of branch impedances and different loading conditions showed that the voltage differences between the two ends of every line/cable segment are small and negligible compared with the infeed point voltage. This is mostly due to the short segment lengths in distribution networks. For example, voltage differences in the 134-bus, 69-bus, and IEEE 37-bus distribution networks are shown in Fig. 2 [18], [31]. The voltage differences for the IEEE 123-bus system are also shown in Section 3. Note that in 
addition to the dominant nonzero values corresponding to the infeed point voltage, the $\ell_{1}$-regularized least-squares problem will identify certain non-zero voltage drops between the two ends of each segment connected to big loads, capacitor banks, DGs, and main three-phase feeders supplying several laterals.
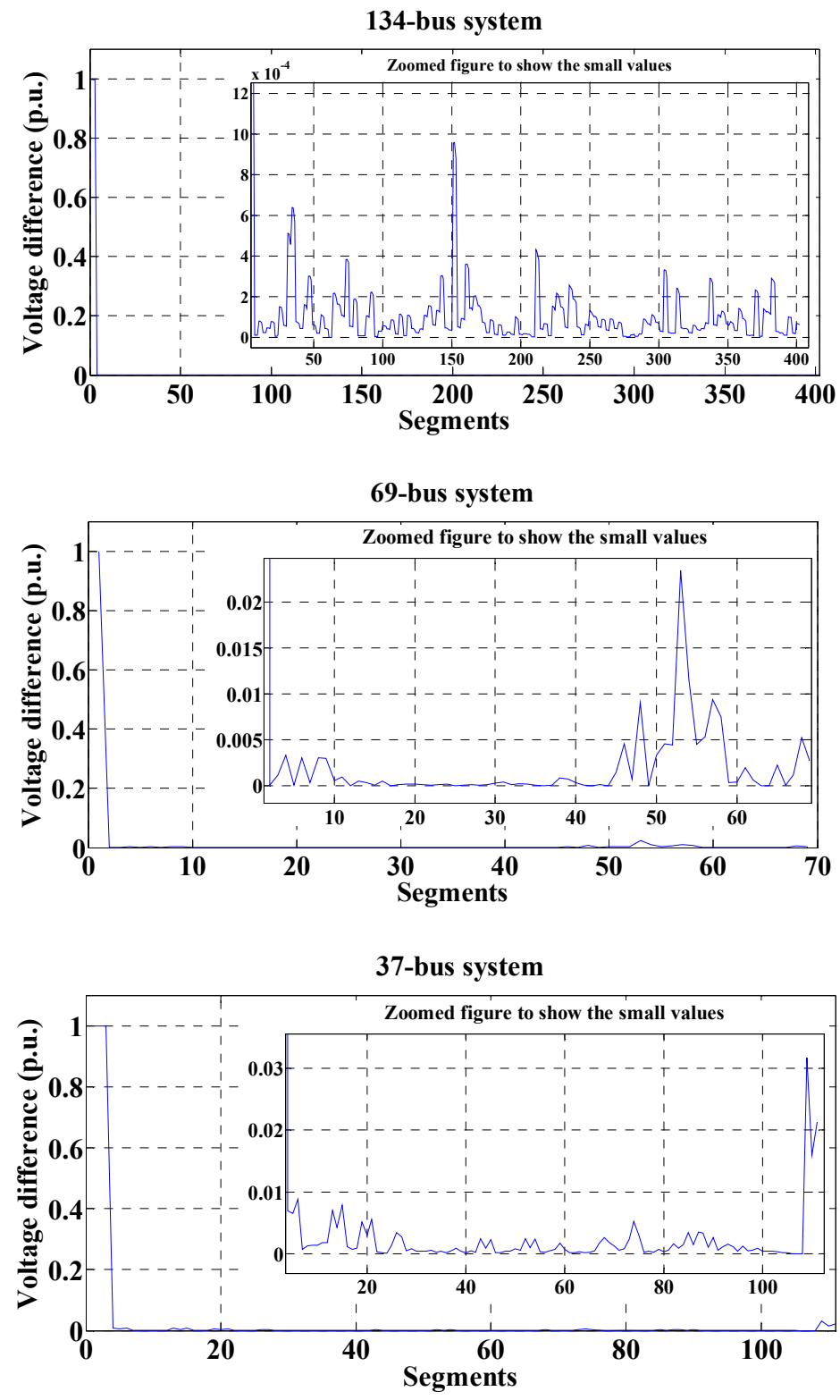

Fig. 2. Voltage differences between two ends of the segments in different distribution networks

The voltage differences under low-load conditions are smaller than the differences for a normal load due to lower segment currents. In order to investigate the voltage drops 
under heavy-load conditions, voltage drops are obtained when loads are scaled up to two, three, and four times the normal values in the IEEE 123-bus test system. The histogram of the voltage drops in all simulations is shown in Fig. 3. As shown, the voltage drops of more than 900 segments in different load conditions are less than 0.002 per unit.

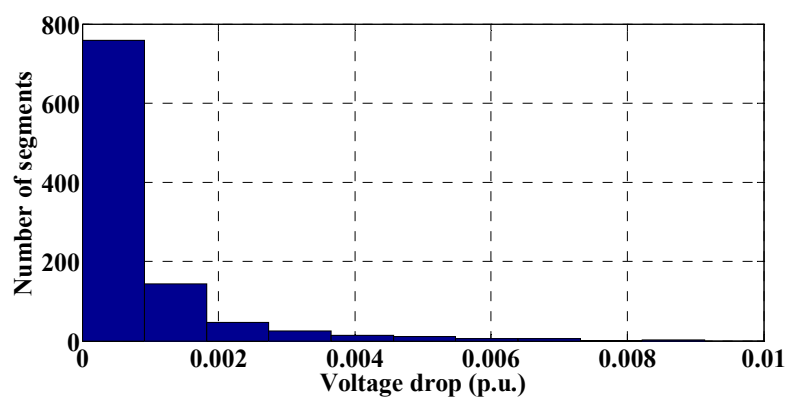

Fig. 3. Histogram of the voltage drops in different load conditions in IEEE 123-bus test system

Voltages at the two ends of any branch are subtracted from each other by a difference transform. If bus 1 is the infeed bus and matrix $(B)$ is the three-phase branch-bus incident matrix, the state vector $\left(x_{0}\right)$ in $(1)$ is sparsified by

$$
S=\left[\begin{array}{cc}
I_{3} & O_{3 \times(n-3)} \\
O_{(n-3) \times 3} & B
\end{array}\right]
$$

For example, the matrix $(S)$ for the 3-bus distribution network in Fig. 1 is

$$
S=\left[\begin{array}{ccc}
I_{3} & O_{3 \times 3} & O_{3 \times 2} \\
I_{3} & -I_{3} & O_{3 \times 2} \\
O_{2 \times 3} & I_{2} \vdots O_{2 \times 1} & -I_{2}
\end{array}\right]
$$

\subsection{Bad Data Processing}

Bad data in voltage measurements are detected, identified and corrected by simultaneously solving the vectors $x_{s}$ and $v$ in (11) based on the joint $\ell_{1}$-minimization method [28]

$$
Z=\overbrace{\left[\begin{array}{ll}
\psi & I_{m}
\end{array}\right]}^{F} \overbrace{\left[\begin{array}{c}
x_{s} \\
v
\end{array}\right]}^{C}
$$


In the absence of bad measurements, the vector $v$ is negligible compared to the vector $x_{s}$. With a few bad measurements, the vector $v$ is sparse with only a few dominant errors corresponding to the bad measurements. Hence, the vector $\mathrm{C}$ is also sparse and recovered by solving an $\ell_{1}$-regularized least-squares problem. The recovered dominant values in the vector $v$ detect and identify the bad measurements. To correct the bad measurements, the recovered errors are subtracted from the bad measured data and (22) is solved with the corrected measurements to recover the vectors $x_{s}$ and $v$. This process is repeated until no dominant value appears in the recovered vector $v$.

Bad data processing steps are as follows:

1- Solve equation (22) to obtain the vectors $x_{s}$ and $v$.

2- If the vector $v$ finds dominant values which are more than the defined noise levels, then there are bad data and large values corresponding to bad measurements and we go to Step 3. Otherwise, stop.

3- Subtract the noise vector $(v)$ from the measurements vector $(Z)$ and go to step 1 .

In our simulations, the bad data algorithm is terminated after 2-3 iterations.

\subsection{Micro-PMUs Placement for Unique Solution}

Optimal $\mu$ PMUs placement along the network is needed to obtain the unique solution of the underdetermined equation (12). The placement algorithm is carried out by satisfying the Karush-Kuhn-Tucker (KKT) optimality conditions for the $\ell_{1}$-regularized least-squares problem [29]

$$
\psi^{T}\left(Z-\psi \cdot x_{s}^{*}\right)=\lambda \cdot \beta, \quad \beta_{i} \in\left\{\begin{array}{c}
\operatorname{sign}\left(x_{s, i}^{*}\right) \text { if } x_{s, i}^{*} \neq 0 \\
{[-1,1] \text { if } x_{s, i}^{*}=0}
\end{array}, \text { for } i=1, \ldots, n\right.
$$

where $\beta \in R^{n}$ is a subgradient of the function $\left\|x_{s}\right\|_{1}$ at the solution $x_{s}^{*}$ of (12). Therefore, $x_{s}^{*}$ is a solution of (12) if and only of $x_{s}^{*}$ satisfies (23) for some $\beta$. The KKT 
conditions give uniqueness conditions for the solution of (12) [29].

Lemma 1 [29]: For any $Z, \psi$ and $\lambda>0$, if null $\left(\psi_{f}\right)=0$, or equivalently $\operatorname{rank}\left(\psi_{f}\right)=$ $f$, then the solution $x_{s}^{*}$ is unique.

In Lemma $1, f$ is the number of nonzero entries in the vector $\left(x_{s}\right)$ and $\psi_{f} \in R^{m \times f}$ is constructed from $f$ columns of $\psi$ corresponding to the nonzero entries of the vector $\left(x_{s}\right)$. Therefore, a priori knowledge of nonzero locations in the sparse vector is required to find the unique solution of (12). In our DSSE algorithm, the dominant nonzero values correspond to the infeed point voltage. Other small nonzero values correspond to the voltage differences between the two ends of each segment connected to big loads, capacitor banks, and DGs. In addition, voltage drops along the main three-phase feeders supplying several laterals are not negligible.

The number of measurements $(m)$ must be at least equal to the number of nonzero values $(f)$ to guarantee that the $\psi_{f}$ is a full column rank matrix. We assume that all $n$ states are monitored with $\mu$ PMUs and form $\psi_{f} \in R^{n \times f}$. The $f$ linearly independent rows of $\psi_{f}$ and their corresponding measurements are then selected by using the permutation matrix through QR factorization. The permutation matrix calculated by QR factorization identifies $f$ linearly independent rows of $\psi_{f}$. The buses corresponding to $f$ selected rows are chosen for sensor placement.

The expected nonzero values due to voltage differences between the two ends of each segment connected to big loads, capacitor banks, DGs, and voltage drops along the main three-phase feeders supplying several laterals may become negligible due to different distribution network loading and operating conditions. Both the zero and nonzero status of these entries are taken into consideration to generate all feasible 
operating conditions. In other words, $\left(\begin{array}{l}f \\ 1\end{array}\right)+\left(\begin{array}{l}f \\ 2\end{array}\right)+\cdots+\left(\begin{array}{l}f \\ f\end{array}\right)$ combinations of nonzero values are considered to cover all possible scenarios. In addition, if other big loads, capacitor banks and DGs are planned for the network, they must also be taken into account. For each possible combination of $\left(\begin{array}{l}f \\ 1\end{array}\right),\left(\begin{array}{l}f \\ 2\end{array}\right), \ldots,\left(\begin{array}{l}f \\ f\end{array}\right)$, the measurement placement is completed by QR factorization and the scenario matrix $\Gamma_{1} \in R^{\zeta \times n_{0}}$ is defined as

$$
\Gamma_{1}(i, j)=\left\{\begin{array}{l}
1, \text { if measurement } j \text { is selected in scenario } i \\
0, \quad \text { Otherwise }
\end{array}\right.
$$

where $\zeta$ is the number of scenarios and $n_{0}$ is the number of phase-to-ground nodes.

Voltage phasors at a bus are measured using $\mu \mathrm{PMU}$ and the voltage phasors at the neighboring buses are estimated using the measured voltage phasors and current flow measurements along the neighboring branches. The bus incidence matrix $\Gamma_{2} \in R^{n_{0} \times n_{0}}$ is defined as

$$
\Gamma_{2}(i, j)=\left\{\begin{array}{l}
1, \text { if node } i \text { is connected to node } j \\
0, \quad \text { Otherwise }
\end{array}\right.
$$

The minimum number of $\mu$ PMUs necessary to provide a unique solution for (12) is obtained by solving a binary integer linear programming

$$
\min \sum_{i=1}^{n_{0}} \mathcal{L}_{i} \text { subject to } \Gamma_{1} \cdot \Gamma_{2} \cdot \mathcal{L}=\mathbf{1}^{\zeta \times 1}, \mathcal{L} \text { is binary }
$$

The $\mu$ PMUs must be installed at the nodes with unity entries in the vector $\mathcal{L} \in R^{n_{0}}$.

\section{SIMULATION RESULTS}

The effectiveness of the proposed DSSE method is verified using the simulation results of the IEEE 123-bus standard unbalanced network, which is modeled in the DIgSILENT Power Factory software [30]. The $\mu$ PMU data are generated by executing 
an unbalanced three-phase load flow and feeding the extracted data to the developed algorithm in MATLAB for DSSE. The accuracy of the estimated states is evaluated by calculating the mean absolute error (MAE), mean squared error (MSE), and two-norm error (TNE) as follows

$$
\begin{gathered}
M A E=\frac{\sum_{i=1}^{n}\left|x_{i}^{\text {estimated }}-x_{i}^{\text {actual }}\right|}{n} \\
M S E=\sqrt{\frac{1}{n} \sum_{i=1}^{n}\left(x_{i}^{\text {estimated }}-x_{i}^{\text {actual }}\right)^{2}} \\
T N E=\sqrt{\sum_{i=1}^{n}\left(x_{i}^{\text {estimated }}-x_{i}^{\text {actual }}\right)^{2}}
\end{gathered}
$$

where the system states $\left(x_{i}\right)$ include the real and imaginary parts of the phase-to-ground voltages and $n$ is double the number of phase-to-ground voltages in the system.

The IEEE 123-bus distribution network is an unbalanced system containing three-, double-, and single-phase branches with 256 phase-to-ground voltages [31]. Using our placement algorithm, eight $\mu$ PMUs are installed at buses $1,13,44,60,65,83,89$, and 101 to provide 86 voltage and current phasors. Using the proposed method, a $\lambda$ value of $10^{-4}$ is obtained. The single line diagram of the test system including the measurement devices is shown in Fig. 4.

Actual and estimated voltages in 256 phase-to-ground nodes are shown in Fig. 5. The MAE, MSE, and TNE of the estimates are $8.017 \times 10^{-5}$ (p. u. ), $2.063 \times 10^{-4}$ (p.u.), and $4.7 \times 10^{-3}$ (p.u.), respectively. These values show that the proposed technique accurately estimates $n=2 n_{0}=2 \times 256=512$ system states using only $m=2 m_{0}=$ $2 \times 84=168$ measurements that are just $32.8 \%$ of the system states. In addition, the actual and estimated sparse representations of the voltage profile are show in Fig. 6 where the sparse representation of the voltage profile includes three significant values 
for the first three nodes and negligible coefficients for other nodes.

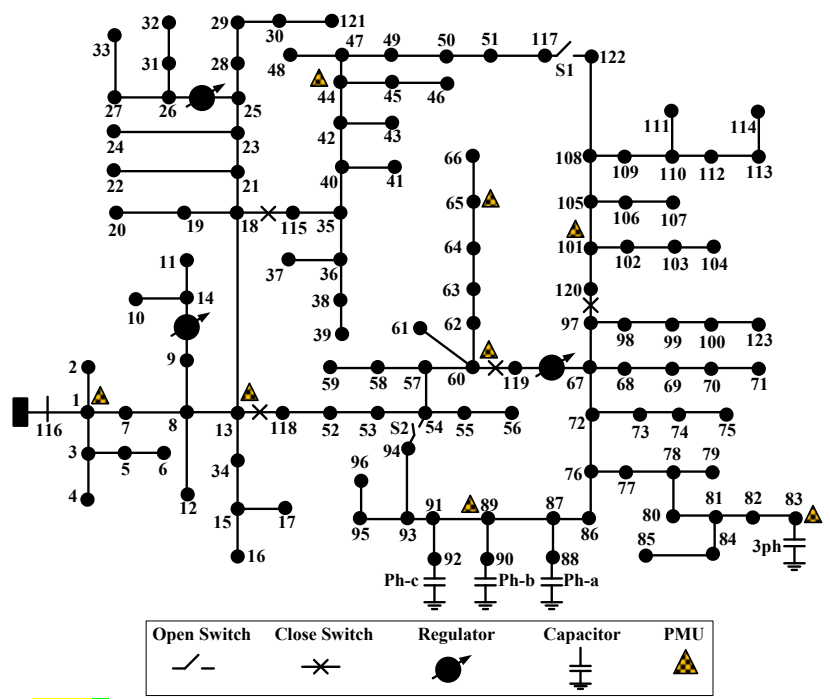

Fig. 4. Single line diagram of the IEEE 123-bus test system

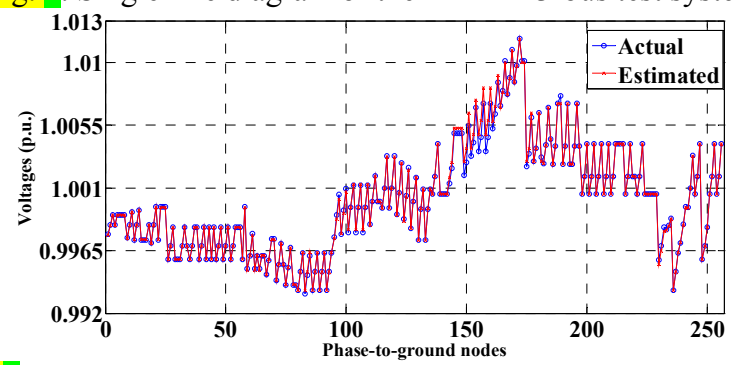

Fig. 5. Actual and estimated voltages in the IEEE 123-bus system

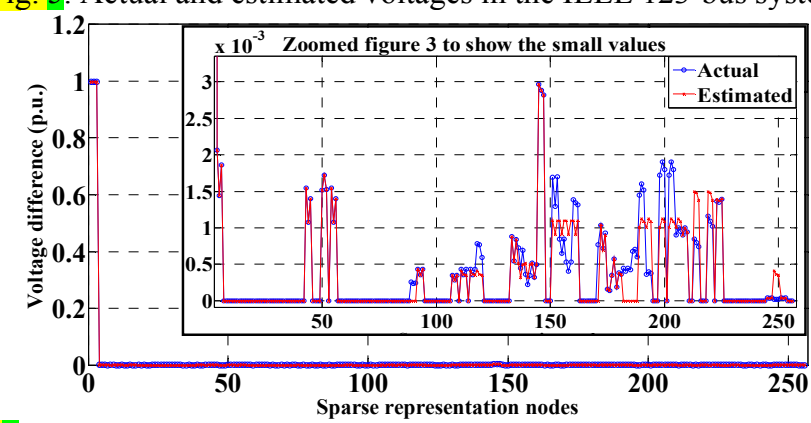

Fig. 6. Actual and estimated sparse representations of the voltage profile

\subsection{Noisy Measurements Assessment}

Since the voltage angle differences between any two nodes of the distribution networks is small, $\mu$ PMUs tend to be more accurate than at the transmission level and provide meaningful measurements of the voltage angle differences [17]. However, $\mu \mathrm{PMU}$ measurements still include communication and transducer errors.

To simulate the measurement errors, we add zero-mean Gaussian noise with $0.1 \%$ 
standard deviation to the simulated voltage magnitudes and $0.05^{\circ}$ to the angles, as in [17].

The DSSE algorithm is executed and the error indices are calculated 100 times with different Gaussian noise patterns to assess the effect of noise on DSSE accuracy. Table I shows the maximum, the minimum and the average TNE, MAE, and MSE values over the 100 simulation runs. The MAE and MSE values versus simulation runs are shown in Fig. 7. Table I and Fig. 7 show that DSSE accuracy remains in the range of $10^{-4}$ in the presence of random measurement errors.

Table I. Performance of the DSSE method for 100 simulation runs with noisy measurements

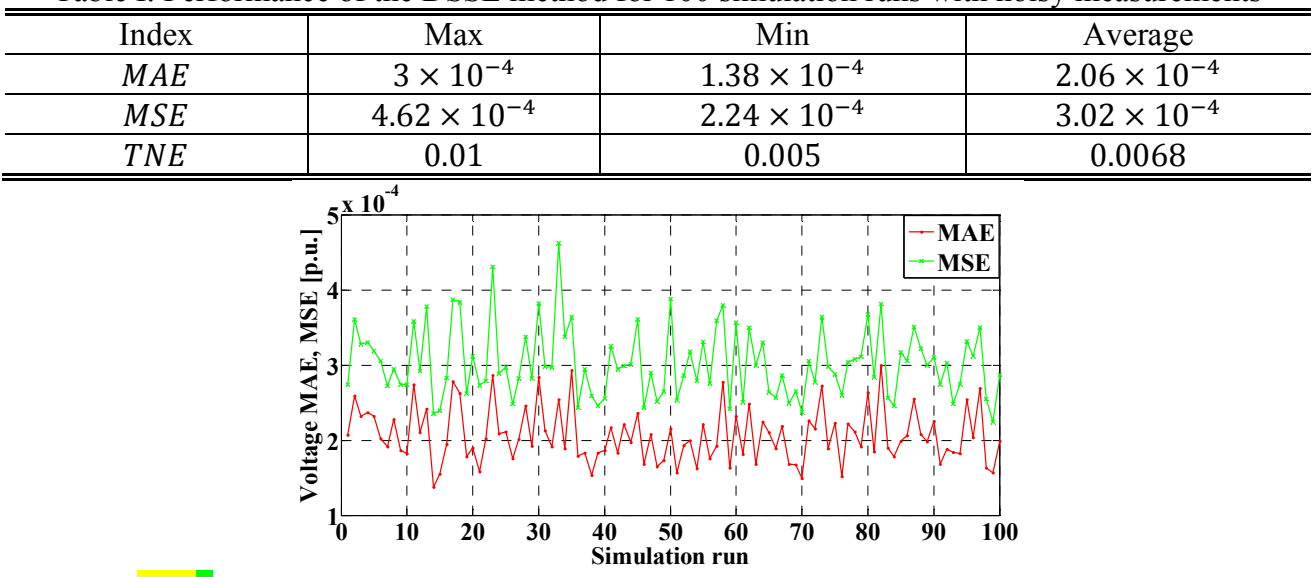

Fig. 7. MAE and MSE values for 100 simulation runs with noisy measurements

\subsection{Noisy and Bad Measurements Assessment}

To examine the impact of bad current measurements on the accuracy of the proposed DSSE, we first add Gaussian noise to all voltage and current measurements. Then we randomly select five current measurements and set two to 0 , multiply one by -0.5 , multiply another by 0.5 , and another by -1 . The simulations are repeated 100 times and the maximum, the minimum, and the mean of the accuracy indices are presented in Table II. The MAE and MSE variations over the 100 simulation runs are shown In Fig. 8. Table II and Fig. 8 show that the DSSE technique is slightly less accurate due to bad current measurements but is still satisfactory. The current magnitudes are much smaller 
than the voltage magnitudes and the errors of the bad current measurements are in the range of the Gaussian noise of the voltage measurements. Hence, the errors of the bad current measurements cannot be reconstructed. However, these errors do not degrade the state estimation accuracy drastically.

Table II. Performance of the DSSE method for 100 simulation runs with noisy measurements and 5 bad current measurements

\begin{tabular}{c|c|c|c}
\hline \hline Index & Max & Min & Average \\
\hline$M A E$ & $5.97 \times 10^{-4}$ & $1.48 \times 10^{-4}$ & $2.99 \times 10^{-4}$ \\
\hline$M S E$ & $11 \times 10^{-4}$ & $2.58 \times 10^{-4}$ & $4.91 \times 10^{-4}$ \\
\hline$T N E$ & 0.024 & 0.0058 & 0.011 \\
\hline \hline
\end{tabular}

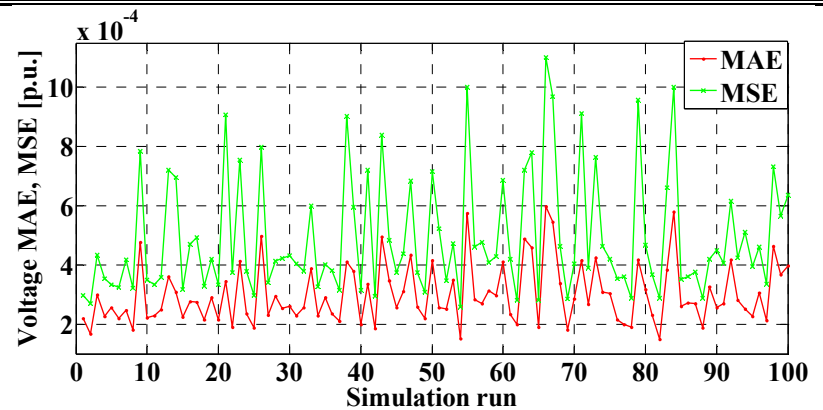

Fig. 8. MAE and MSE values for 100 simulation runs with noisy measurements and 5 bad current measurements

To investigate the performance of the proposed DSSE algorithm with bad voltage measurements, the system states are estimated when all voltage and current measurements are corrupted by Gaussian noise and up to three voltage measurements are multiplied by 0.95 and 1.05. Our simulations do not include bad voltage measurements that are zero, negative, negligible, or excessively large because such bad data are easily removed in plausibility checks. Our bad data processing algorithm detects, identifies and corrects one hundred random combinations of single, double, and triple bad voltage measurements. The average accuracy indices (i.e. MAE, MSE, and TNE) over 100 simulation scenarios with and without bad voltage correction are presented in Table III. The correction step removes large noise from the bad voltage measurements and enhances state estimation accuracy. 
Table III. Performance of the DSSE method for 100 simulation runs with and without the bad voltage correction

\begin{tabular}{c|c|c}
\hline \hline \multirow{2}{*}{ Index } & \multicolumn{2}{|c}{ Average } \\
\cline { 2 - 3 } & With correction & Without correction \\
\hline$M A E$ & $2.65 \times 10^{-4}$ & $9 \times 10^{-3}$ \\
\hline$M S E$ & $3.64 \times 10^{-4}$ & $1.77 \times 10^{-2}$ \\
\hline$T N E$ & 0.009 & 0.4 \\
\hline \hline
\end{tabular}

The differences between the erroneous measurements and estimated values, called residual values, are shown in three scenarios with one, two and three bad voltage measurements in Fig. 9. There are 8 three-phase voltage measurements in the system capturing 24 phase-to-ground voltages.
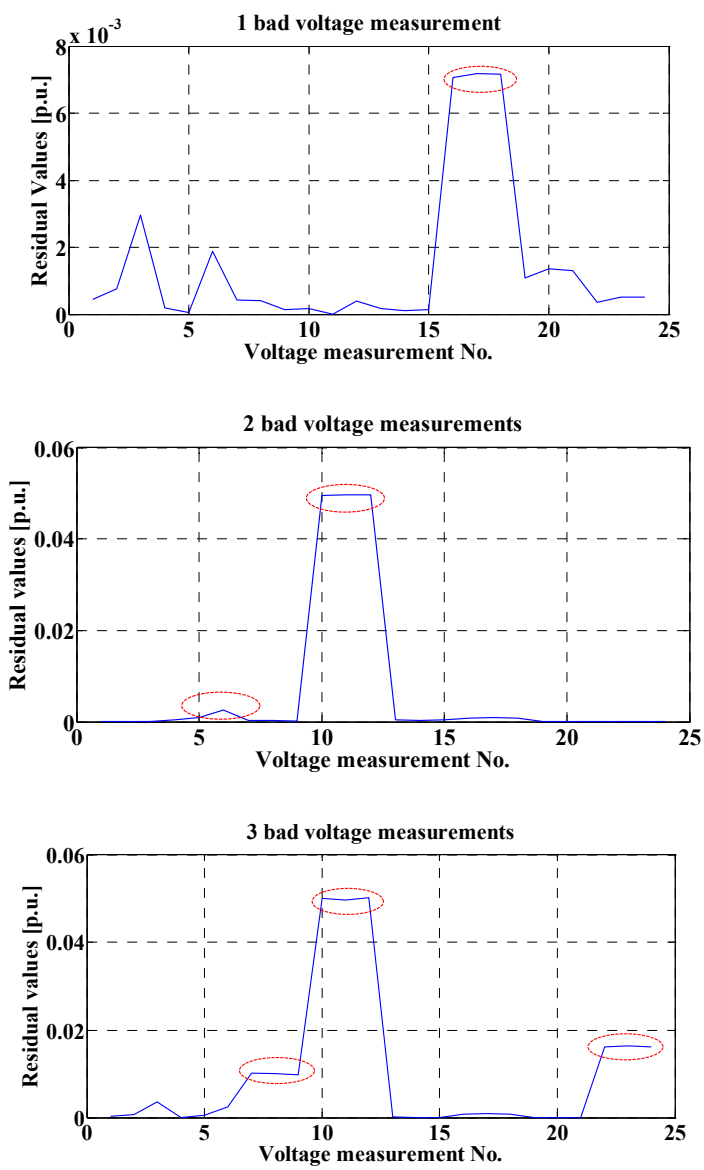

Fig. 9. Residual values of voltage measurements in different bad voltage scenarios

In order to clarify the bad data processing steps, an illustrative example is presented 
here. Suppose that there exist two bad voltage measurements in the system at buses 83 and 101. One voltage is multiplied by 0.95 and another is multiplied by 1.05 . The vectors $x_{s} \in R^{256}$ (voltage magnitudes and angles) and $v \in R^{84}$ (24 voltage measurement noises and 60 current measurement noises) are estimated by solving (22). The estimated and actual voltage magnitudes and estimated voltage measurements noises are shown in Fig. 10.
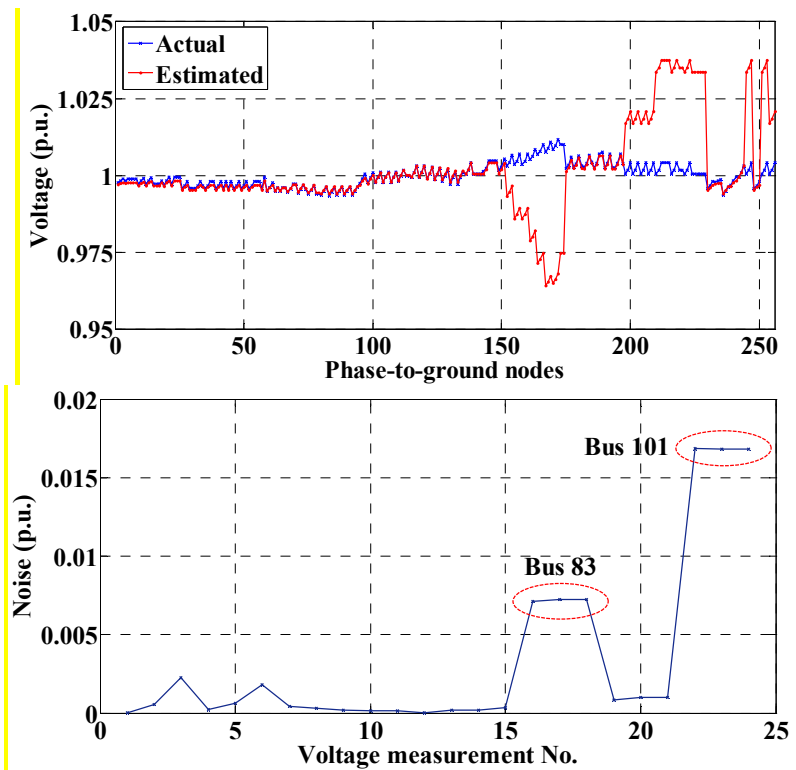

Fig. 10. Estimated and actual voltage magnitudes and estimated voltage measurements noises with two bad voltage measurements

Since voltage measurements are contaminated with $0.1 \%$ noise level which is almost equivalent with 0.001 p.u., those two measurements, which are within the noise level of $0.01 \mathrm{pu}$, are identified as the bad data. The estimated noise vector $(v)$ is subtracted from the measurements vector $(Z)$ and the bad data process is repeated for two iterations. The estimated voltages and noise magnitudes after the denoising process are shown in Fig. 11. 

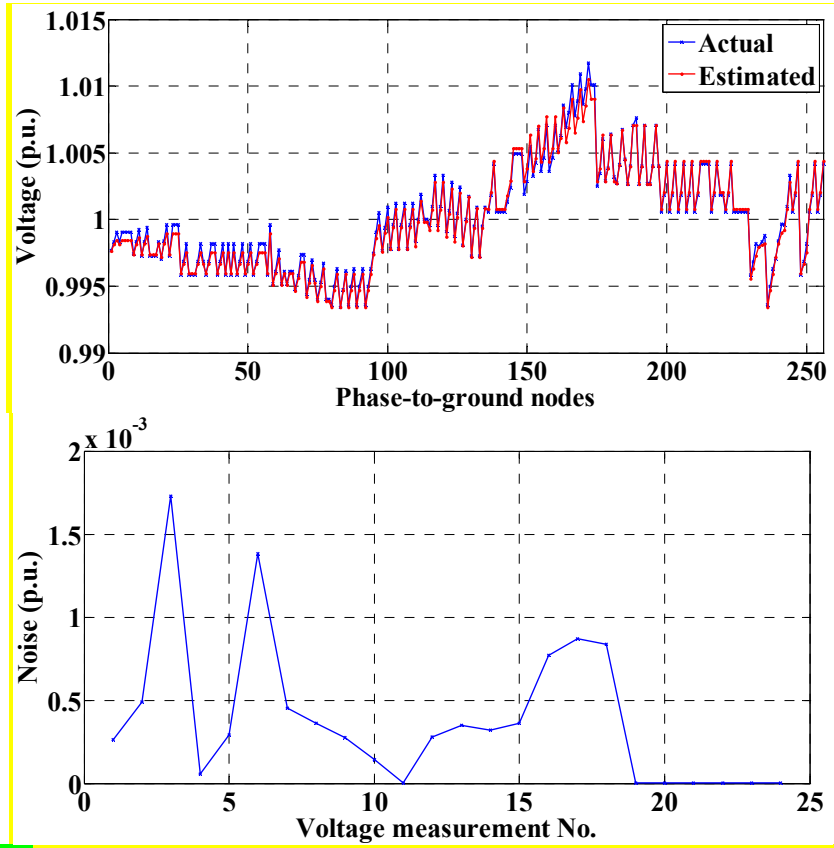

Fig. 11. Estimated voltages and noise magnitudes after the denoising process

The joint $\ell_{1}$-minimization method performs well even with low accuracy measurements. In order to demonstrate the denoising performance of the method, all voltage measurements are corrupted with Gaussian noise with 1\%, 2\%, and 3\% standard deviation and all angle measurements with $0.5^{\circ}, 1^{\circ}$, and $1.5^{\circ}$ (ten, twenty, and thirty orders of magnitude larger than the standard errors of the $\mu$ PMUs) [17]. Fig. 12 shows the MSEs over the 40 simulations with and without the denoising.
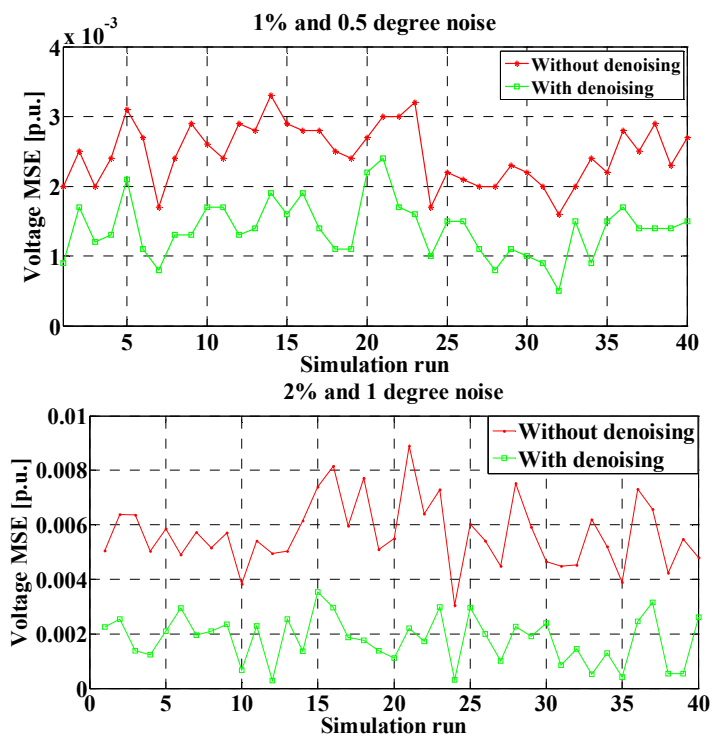


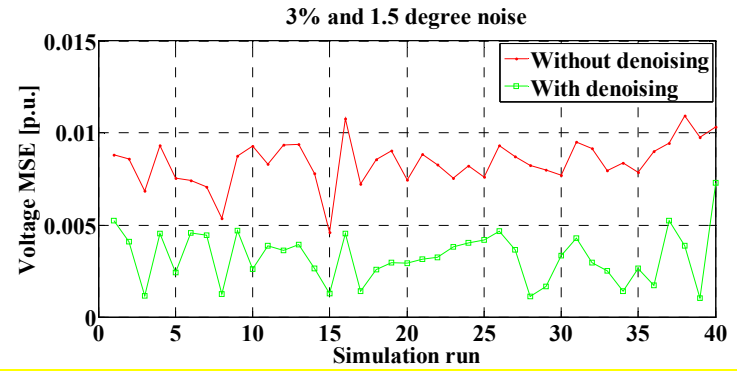

Fig. 12. MSE values for 40 simulation runs with and without the denoising process

\subsection{DGs and Meshed Network Assessment}

The performance of the proposed DSSE is investigated when some DGs are added to the test system and switches are closed to change the radial system to a meshed network. The DSSE accuracy is tested in two arbitrary scenarios as follows:

Scenario 1: Two 1 MW DGs with $\mathrm{PF}=0.8$ are connected at buses 35 and 56 and switch 1 connects the bus 117 to 122 .

Scenario 2: Four $1 \mathrm{MW}$ DGs with $\mathrm{PF}=0.8$ are connected at buses $35,56,66$, and 21 and switch 1 connects the bus 117 to 122 and switch 2 connects the bus 54 to 94 .

The maximum, minimum, and average of the accuracy indices (i.e. $M A E, M S E$, and TNE) over 100 simulation runs for each scenario are presented in Table IV. The proposed DSSE method performs well in the presence of DGs connected to the network operated in a meshed mode. All the simulations scenarios are executed on a computer with a 2.4-GHz CPU and 4.00-GB memory. The DSSE algorithm requires 3 to $5 \mathrm{~s}$ to estimate the system states. Note that no special effort is made for code optimization.

Table IV. Performance of the DSSE method with DGs in the meshed operation mode for 100 simulation runs

\begin{tabular}{c|c|c|c|c}
\hline \hline Scenario & Index & Max & Min & Average \\
\hline \multirow{4}{*}{1} & $M A E$ & $3.83 \times 10^{-4}$ & $1.8 \times 10^{-4}$ & $2.59 \times 10^{-4}$ \\
\cline { 2 - 5 } & $M S E$ & $5.04 \times 10^{-4}$ & $3.18 \times 10^{-4}$ & $3.88 \times 10^{-4}$ \\
\cline { 2 - 5 } & $T N E$ & 0.011 & 0.007 & 0.008 \\
\hline \multirow{3}{*}{2} & $M A E$ & $4.34 \times 10^{-4}$ & $2.1 \times 10^{-4}$ & $2.59 \times 10^{-4}$ \\
\cline { 2 - 5 } & $M S E$ & $5.62 \times 10^{-4}$ & $3.72 \times 10^{-4}$ & $3.88 \times 10^{-4}$ \\
\cline { 2 - 5 } & $T N E$ & 0.013 & 0.007 & 0.01 \\
\hline \hline
\end{tabular}




\section{DISCUSSION AND COMPARISONS}

In this section, we discuss the most important results of our paper and compare their performance to results from the literature. Unlike this paper, the method in [16] requires a large numbers of smart meters with the PMU functionality. Also, the performance of the proposed algorithm in [16] is not evaluated when the measured data are contaminated with bad data. In [14], the asynchronicity of SM measurements is taken into account to make the SM data more realistic for WLS-based DSSE methods. In [14], the average TNE of the estimated states is about 0.08 for the IEEE 13-bus test system. Our DSSE algorithm results in an average TNE of 0.0068 for the IEEE 123-bus test system. Thus, our approach of minimizing the $\ell_{1}$-norm of the compressed voltage profile using a few accurate $\mu$ PMUs performs better than WLS-based DSSE that minimizes squared measurement errors using a large number of SMs with lower accuracy. Distribution networks with different levels of monitoring infrastructure can benefit from the proposed DSSE algorithm. If the network is not equipped with any SMs, the proposed $\mu$ PMU-based DSSE is a more economical option.

$\mu$ PMUs accurately record the sampling time of measurements by the global positioning system (GPS) and frequently transmit the measurements to the monitoring and control center via Ethernet. While the reporting intervals between SM and SCADA measurements are on the order of minutes, $\mu$ PMUs transfer the data to the data concentrators which communicate with the server every few seconds. If one distribution network is already fully observable with SMs, our $\mu$ PMU-based DSSE technique can be separately executed more frequently to accurately estimate the state of system snapshots between two sequential runs of SM-based DSSE [32]. 
In addition, two sets of voltage magnitudes and angles that are estimated by classical WLS methods using SM/SCADA measurements and the proposed $\mu$ PMU-based method can be combined together to accurately re-estimate the system states in a linear post-processing step. Existing post processing studies only combine a few voltage magnitudes and angles measured by PMUs with one set of voltage magnitudes and angles estimated from classical DSSE methods [33].

In this approach, the estimated states by the proposed DSSE algorithm $U_{1}^{*} \in R^{3 N}$ and the estimated states by classic WLS-based state estimators $\mathrm{U}_{2}^{*} \in \mathrm{R}^{3 \mathrm{~N}}$ are used to provide a linear post-processing system to re-estimate the system states

$$
\begin{aligned}
{\left[\begin{array}{l}
U_{1}^{*} \\
U_{2}^{*}
\end{array}\right] } & =\left[\begin{array}{l}
I_{3 N} \\
I_{3 N}
\end{array}\right] U+\left[\begin{array}{l}
e_{1}^{*} \\
e_{2}^{*}
\end{array}\right] \\
Z_{1} & =H_{1} \cdot U+e_{1}
\end{aligned}
$$

where $I$ is the identity matrix, $Z_{1} \in R^{6 N}$ is the estimated complex voltage phasors, $H_{1} \in R^{6 N \times 3 N}$ is the constant Jacobian matrix, and $e_{1} \in R^{6 N}$ is the error vector of estimated states. Equation (31) is solved by the WLS method to re-estimate the system states vector $U \in R^{3 N}$ whose accuracy is significantly improved compared to the $U_{2}^{*}$ accuracy.

Furthermore, hybrid state estimators can incorporate the voltage magnitudes and angles $\left(U_{1}^{*}\right)$ estimated from the proposed $\mu$ PMU-based method into SM measurements including active/reactive power measurements in their iterative least-squares process to enhance the SE accuracy [34]

$$
\left[\begin{array}{c}
U_{1}^{*} \\
z
\end{array}\right]=\left[\begin{array}{c}
I_{3 N} \cdot U \\
h(|U|, \measuredangle U)
\end{array}\right]+\left[\begin{array}{c}
e_{1}^{*} \\
e_{z}
\end{array}\right]
$$

where $z$ is the active/reactive power measurement vector, $h($.$) is a nonlinear function$ relating power measurements to state variables and $e_{z}$ is the vector of power 
measurement errors. The sampling times of $\mu$ PMUs are usually reported accurately. However, SM data sampling time may include some errors. In order to synchronize these two data, larger error terms for SM/SCADA data can be considered as discussed in [14].

The estimated voltage magnitudes and angles by our $\mu$ PMU-based method can accurately yield synchronized pseudo measurements and synchronize the SM values. These data significantly improve the performance of the WLS-based DSSE algorithm. These new directions and alternative approaches provide a broad range of research topics to improve the performance of classical DSSE methods using our proposed $\mu$ PMU-based DSSE.

\section{CONClusions ANd Future Works}

This paper introduces a novel three-phase distribution system state estimation (DSSE) paradigm exploiting compressive sensing $(\mathrm{CS})$ and $\ell_{1}$ regularization to estimate the system states from a limited number of micro-phasor measurement units ( $\mu$ PMUs). The proposed technique does not require pseudo measurements (e.g. smart meter data) and system observability. The effectiveness of the proposed method with noisy measurements, different distributed generation (DG) penetration levels, and meshed operation modes is verified by simulation results. In addition, bad current measurements do not notably influence the accuracy of the proposed DSSE technique. Joint $\ell_{1}$ minimization effectively detects, identifies and corrects bad voltage measurements. The estimation errors of the proposed technique are at least an order of magnitude smaller than those in the WLS-based DSSE algorithm using smart meter data. As the future works, we will develop a hybrid DSSE by incorporating $\mu$ PMU data into the WLS- 
based technique. Furthermore, we will develop an integrated two-step SE utilizing the output of the $\mu$ PMU-based SE and smart meter data.

\section{REFERENCES}

[1] D. A. Haughton and G. T. Heydt, "A linear state estimation formulation for smart distribution systems,” IEEE Trans. Power Syst., vol. 28, no. 2, pp. 1187-1195, May 2013.

[2] I. Roytelman and S. M. Shahidehpour, "State estimation for electric power distribution system in quasi real-time conditions," IEEE Trans. Power Del., vol. 8, no. 4, pp. 2009-2015, Oct. 1993.

[3] M. E. Baran and A. W. Kelley, "State estimation for real-time monitoring of distribution systems," IEEE Trans. Power Syst., vol. 9, no. 3, pp. 1601-1609, Aug. 1994.

[4] M. E. Baran and A. W. Kelley, "A branch-current-based state estimation method for distribution systems," IEEE Trans. Power Syst., vol. 10, no. 1, pp. 483-491, Feb. 1995.

[5] C. N. Lu, J. H. Teng, and W.-H. E. Liu, "Distribution system state estimation," IEEE Trans. Power Syst., vol. 10, no. 1, pp. 229-240, Feb. 1995.

[6] W.-M. Lin and J.-H. Teng, "Distribution fast decoupled state estimation by measurement pairing," IEE Proc. Gener. Transm. Distrib., vol. 143, no. 1, pp. 43-48, Jan. 1996.

[7] W.-M. Lin and J.-H. Teng, "State estimation for distribution systems with zero-injection constraints," IEEE Trans. Power Syst., vol. 11, no. 1, pp. 518-524, Feb. 1996.

[8] D. L. Lubkeman, J. Zhang, A. K. Ghosh, and R. H. Jones, "Field results for a distribution circuit state estimator implementation," IEEE Trans. Power Del., vol. 15, no. 1, pp. 399-406, Jan. 2000.

[9] K. Li, "State estimation for power distribution system and measurement impacts," IEEE Trans. Power Syst., vol. 11, no. 2, pp. 911-916, May 1996.

[10] A. K. Ghosh, D. L. Lubkeman, M. J. Downey, and R. H. Jones, "Distribution circuits state estimation using a probabilistic approach," IEEE Trans. Power Syst., vol. 12, no. 1, pp. 45-51, Feb. 1997.

[11]E. Manitsas, R. Singh, B. C. Pal, and G. Strbac, "Distribution system state estimation using an artificial neural network approach for pseudo measurement modeling," IEEE Trans. Power Syst., vol. 27, no. 4, pp. 1888-1896, Nov. 2012.

[12] V.Miranda, J. Pereira, and J. T. Saraiva, "Load allocation in DMS with a fuzzy state estimator," IEEE Trans. Power Syst., vol. 15, no. 2, pp. 529-534, May 2000.

[13] B.P.Hayes, J.K.Gruber, M.Prodanovic, "A Closed-Loop State Estimation Tool for MV Network Monitoring and Operation," IEEE Trans. Smart Grid, Early Access.

[14] A.Alimardani, F.Therrien, D.Atanackovic, J.Jatskevich, and E.Vaahedi, "Distribution System State Estimation Based on Nonsynchronized Smart Meters," IEEE Trans. Smart Grid, Early Access.

[15] K. Samarakoon, W. Jianzhong, J. Ekanayake, and N. Jenkins, "Use of delayed smart meter measurements for distribution state estimation," in Proc. IEEE Power Energy Soc. Gen. Meeting, San Diego, CA, USA, 2011, pp. 1-6.

[16] J.B.Leite, J.R.S.Mantovani, "Distribution System State Estimation Using the Hamiltonian Cycle Theory," IEEE Trans. Smart Grid, Early Access.

[17] A. von Meier, D. Culler, A. McEachen, and R. Arghandeh, "Micro-synchrophasors for distribution systems," in Proceedings of IEEE Innovative Smart Grid Technologies Conference (ISGT2014), 2014.

[18] M. Majidi, A. Arabali, and M. Etezadi-Amoli, "Fault Location in Distribution Networks by Compressive Sensing” IEEE Trans. Power Del., vol. 30, no. 4, pp. 1761-1769, Aug. 2015.

[19] M. Majidi, M. Etezadi-Amoli, and M. S. Fadali, "A Novel Method for Single and Simultaneous Faults Location in Distribution Networks" IEEE Trans. Power Syst., vol. 30, no. 6, pp. 3368-3376, Nov. 2015.

[20] K.Vassilis and Georgios Giannakis, "Distributed robust power system state estimation," IEEE Trans. Power Syst., vol 28, no. 2, pp. 1617-1626, 2013.

[21] R.Jalilzadeh Hamidi, H. Khodabandehlou, H. Livani, M.Sami Fadali, “Application of distributed Compressive Sensing to Power System State Estimation," North American Power Symposium (NAPS), Charlotte, NC, USA, 2015, pp. 1-6.

[22] M. Gol and A. Abur, "A Fast Decoupled State Estimator for Systems Measured by PMUs," IEEE Trans. Power Syst., vol. 30, no, 5, pp. 2766-2771, Sep. 2015.

[23] A. Neumaier, "Solving ill-conditioned and singular linear systems: A tutorial on regularization," SIAM Rev., vol. 40, no. 3, pp. 636-666, 1998. 
[24] S. J. Kim, K. Koh, M. Lustig, S. Boyd, and D. Gorinevsky, “An Interior-Point Method for LargeScale 1'-Regularized Least Squares," IEEE Journal on Selected Topics in Signal Processing, vol.1, no.4, pp.606-617, December 2007.

[25]D. L. Donoho, “Compressed sensing,” IEEE Trans. Inform. Theory, vol. 52, pp. 1289-1306, Sept. 2006.

[26]E. Candès and J. Romberg, I -magic: A Collection of MATLAB Routines for Solving the Convex Optimization Programs Central to Compressive Sampling 2006 [Online]. Available: www.acm.caltech.edu/l1magic/

[27] The MOSEK Optimization Tools Version 2.5. User's Manual and Reference 2002 [Online]. Available: www.mosek.com, MOSEK ApS

[28] J. Wright and Y. Ma, "Dense error correction via 11-minimization," in ICASSP 2009.

[29] R. J. Tibshirani et al., "The Lasso problem and uniqueness," Electron. J. Statist., vol. 7, pp. 14561490, 2013.

[30]DIgSILENT, [online] Availabe: http://www.digsilent.de/

[31][Online]Available:http://ewh.ieee.org/soc/pes/dsacom/testfeeders/index.html

[32] M.Pignati, M.Popovic, S Barreto, and et al., "Real-Time State Estimation of the EPFL-Campus Medium-Voltage Grid by Using PMUs," in Proc. the Sixth Conference on Innovative Smart Grid Technologies (ISGT2015), Washington, DC, USA, February 17-20, 2015.

[33] J. Liu, J. Tang, F. Ponci, A. Monti, C. Muscas, and P. A. Pegoraro, "Trade-offs in pmu deployment for state estimation in active distribution grids,” IEEE Trans. Smart Grid, vol. 3, no. 2, pp. 915-924, Jun. 2012.

[34] M. Pau, P. A. Pegoraro, and S. Sulis, "Efficient branch-current-based distribution system state estimation including synchronized measurements," IEEE Trans. Instrum. Meas., vol. 62, no. 9, pp. 2419-2429, Sep. 2013.

\section{ACKNOWLEDGeMENT}

This paper is based upon work supported by the National Science Foundation under

Grant No. IIA-1301726. 